\title{
Manufacturing risk management in optical design
}

\author{
O.Faehnle1 and I.Livshits2 \\ ${ }^{1}$ OST-University of Applied Sciences, Buchs, Switzerland, \\ ${ }^{2}$ ITMO University, St. Petersburg, Russia
}

\begin{abstract}
This paper reports on strategies to determine optimal sets of optical fabrication technologies (OFT) for given optical elements, to be applied in optical fabrication chains. For this purpose, optical systems are categorized and linked to critical characteristics of optical fabrication technologies, which are determined by a methodical analysis of their processing parameters.
\end{abstract}

\section{Introduction}

Predictable and stable fabrication processes are essential for reliable cost and quality management in optical fabrication technology. This paper describes strategies to determine for given optical elements and systems optimum sets of optical fabrication technologies (OFT) to be applied in optical fabrication chains.

\section{Optical systems generation and classification}

Mankind has been applying light as a tool to solve daily life challenges such as detection, illumination and information transmittance. And to apply light as a tool, it takes optical systems. Consequently, optical systems, e.g., objectives, interferometers, optical computer mice, endoscopes, telescopes, or lasers, are good examples of today's light tools we apply to meet essential needs with which we are confronted. During the generation of optical systems (composed of various optical elements properly mounted and functioning together), three authorities are sequentially engaged: "optical systems design," followed by "optical fabrication design" and finally its actual "manufacturing" (see Table 1).

It is the job of "optical designers" to translate customers' requirements into a well-toleranced optical system design comprising various optical elements (such as lenses, mirrors, and beam splitters) modulating the traversing light as desired.

Subsequently, it is the "optics fabrication designer" who translates the specifications of the finished optical system design and tolerances of the employed optical elements into optimized fabrication chains consisting of a series of subsequent fabrication steps taking available machinery and technologies into account ensuring optimum performance at minimum manufacturing costs. Furthermore, systems stability and durability as well as safety of manufacturers, the eventual users and the environment have to be taken into consideration. In the optimized fabrication chain, each fabrication step (e.g., polishing ${ }^{1}$ ) employs a specific optical fabrication process (e.g., bonnet polishing, ${ }^{2}$ bowl feed polishing, ${ }^{3}$ magnetorheological finishing, ${ }^{4}$ ion beam figuring, ${ }^{5}$ ultraform finishing, ${ }^{6}$ single-point diamond turning, ${ }^{7}$ and fluid jet polishing ${ }^{8}$ ) the choice of which depends on the right balance between quality, fabrication cost, and number of systems to be produced.
Finally, the optical system is being manufactured, applying the developed fabrication chain and processes not exceeding the designed tolerances generating a fully functioning light tool that meets customers' demands. Optical systems can be classified applying different strategies, a.o. by the markets they are applied to, e.g. astronomy, medical, lighting or lithography, or by the type of instruments they are applied in, e.g. microscopes, telescopes, endoscopes or interferometers.

But to be able to identify for a given optical element the optimum optical fabrication technologies, it is important to characterize optical elements by their four major characteristics relevant for fabrication, which are summarized in table2.

\section{Optical fabrication technologies classification}

While a limited number of optics fabrication technologies have been around for millenniums, their diversity level has been increasing tremendously since the first industrial production was started 1670 for Leeuwenhoek's microscope in Delft and due to the increasing demands in application and quality ever since (see Fig.1). Consequently, today there are more than 300 optical fabrication technologies existing, making the optimum choice for the generation of a given optical element a challenge.

Analyzing the optical fabrication technologies methodologically, we identify that they are based on only about 11 polishing techniques: Fresh feed polishing (FFP), ductile machining (DG), chemical polishing (CP), bowl feed polishing (BFP), elastic emission machining (EEM), magnetorheological finishing (MRF), laser fire polishing (LP), ion beam figuring (IBF), abrasive slurry jet machining (ASJ), plasma assisted chemical etching (PACE) as well as laser-induced backside wet etching (LIBWE).

If we analyze the interaction between these different polishing techniques and surfaces being processed, we identify 5 different wear approaches as shown in Fig,2 which are based on (i) 2-body abrasion, (ii) 3body abrasion, (iii) kinetic abrasion, (iv) energy transmission and (v) chemical reaction. Each of them featuring specific strong and weak characteristics in terms of performance and applicability (indicated by “+” and “-“ in Fig.2.). Within these 5 wear approaches various combinations of five different wear processes are possible based on (see Fig.3) (a) brittle cracking, (b) 
ductile flow, (c) chemical reactions, (d) heat and (e) sputtering.

Taking the above into account, it is possible to classify the 11 existing polishing techniques in terms of wear approaches possible and combinations of wear processes they exist of, as shown in table3.

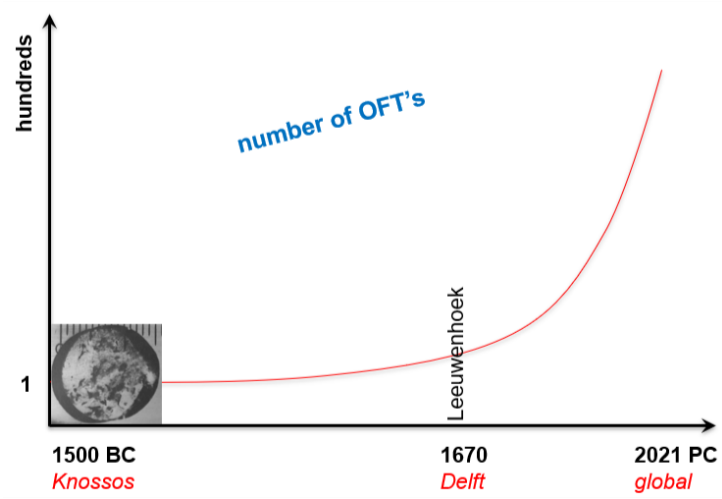

Fig.1. Sketch of development of number of optical fabrication technologies starting from ancient lenses found on Crete through 1670 the first industrial optical fabrication until today.

\section{Matching optical elements with optical fabrication technologies}

Taking the four key characteristics of optical elements into accout (see table2) and extending the methodological classification of polishing techniques (summarized in table3) towards e.g. characteristics of machining kinematics and types of tool-to-workpiece contacts (e.g. point, line and area contacting), enables the classification and modeling of optical fabrication technologies.

Consequently, a data based optimum selection of optical fabrication technologies for given optical elements can be carried out already during the design phase of optical systems generation.
Fig.3 and 4 show, as an example given, an application of the methodological analysis of optical fabrication technologies to identify optimum fabrication chains for a spherical lens, minimizing production risk.

\section{References}

[1] Cook, Lee M. "Chemical processes in glass polishing." Journal of non-crystalline solids 120.1, pp. 152-171, 1990;

[2] David Walker ; A. T. Beaucamp ; David Brooks ; Richard Freeman ; Andrew King ; Gerry McCavana ; Roger Morton ; David Riley ; John Simms; Novel CNC polishing process for control of form and texture on aspheric surfaces. Proc. SPIE 4767, Current Developments in Lens Design and Optical Engineering III, 2002;

[3] J.van Wingerden, H.J. Frankena and B.A. van der Zwan, "Production and measurement of superpolished surfaces", Optical Engineering 31, 1086-1092, 1992;

[4] William I. Kordonski and Stephen D. Jacobs, "Magnetorheological finishing", Int. J. Mod. Phys. B 10, 2837, 1996;

[5] Franz Thomas, and Thomas Hänsel, "Ion beam figuring (IBF) solutions for the correction of surface errors of small high performance optics." Optical Fabrication and Testing. Optical Society of America, 2008;

[6] Edd Fess ; J. Schoen ; M. Bechtold ; D. Mohring, "UltraForm finishing", Proc. SPIE 5786, Window and Dome Technologies and Materials IX, 305, doi:10.1117/12.606468, 2005;

[7] Jiwang Yan, J. Tamaki, K. Syoji, T. Kuriyagawa, "Single-point diamond turning of $\mathrm{CaF} 2$ for nanometric surface", The International Journal of Advanced Manufacturing Technology, Volume 24, Issue 9, pp 640-646, 2004

[8] O. Fähnle, H. van Brug and H. Frankena, "Fluid Jet Polishing of optical surfaces", Applied Optics 37(28), 6771-6773, 1998;

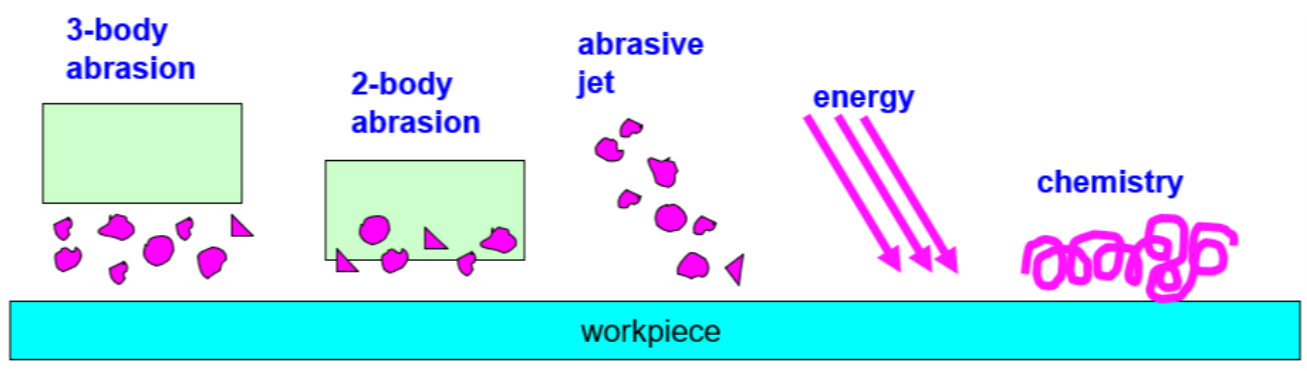

\begin{tabular}{|c|c|c|c|c|c|}
\hline - & $\begin{array}{c}\text { mech.contact, } \\
\text { slow, tool wear }\end{array}$ & $\begin{array}{c}\text { mech.contact, } \\
\text { MSF }\end{array}$ & $\begin{array}{c}\text { material density } \\
\text { dependency }\end{array}$ & $\begin{array}{c}\text { temperature, } n, \\
\text { vapour, } \\
\text { material stress }\end{array}$ & $\begin{array}{c}\text { type ofreactions, } \\
\text { residuals, material } \\
\text { stress }\end{array}$ \\
\hline+ & highly accurate & $\begin{array}{c}\text { fast, } \\
\text { little tool wear }\end{array}$ & $\begin{array}{c}\text { non-contact, } \\
\text { highly accurate }\end{array}$ & $\begin{array}{c}\text { non-contact, } \\
\text { highly accurate }\end{array}$ & $\begin{array}{c}\text { non-contact, } \\
\text { uniform }\end{array}$ \\
\hline
\end{tabular}

Fig.2. Optical polishing techniques are applied in five different wear approaches, each with its strong ("+") and weak ("-“") characteristics in terms of generating optical elements. 


\begin{tabular}{|c|l|l|}
\hline & \multicolumn{1}{|c|}{ Task } & \multicolumn{1}{c|}{ Result } \\
\hline customer & $\begin{array}{l}\text { definition of requirements for light to be } \\
\text { used as a tool ("light tool") }\end{array}$ & $\begin{array}{l}\text { light tool specifications (e.g. MTF, } \\
\text { image resolution, dB, LIDT) }\end{array}$ \\
\hline $\begin{array}{c}\text { optical system } \\
\text { designer }\end{array}$ & $\begin{array}{l}\text { translation of product specifications into a } \\
\text { well toleranced optical system design }\end{array}$ & $\begin{array}{l}\text { well toleranced and specified } \\
\text { optical system design }\end{array}$ \\
\hline $\begin{array}{c}\text { optical } \\
\text { fabrication } \\
\text { designer }\end{array}$ & $\begin{array}{l}\text { translation of optical system design into } \\
\text { fabrication chain and fabrication processes } \\
\text { parameters }\end{array}$ & $\begin{array}{l}\text { fabrication chain and fabrication } \\
\text { process parameters defined and } \\
\text { documented (including measuring } \\
\text { technology) }\end{array}$ \\
\hline $\begin{array}{c}\text { manufacturing } \\
\text { department }\end{array}$ & $\begin{array}{l}\text { manufacturing of optical system as designed } \\
\text { by employing skilled employees and } \\
\text { applying optimized machines at high levels } \\
\text { of automation. }\end{array}$ & $\begin{array}{l}\text { optical system (light tool); ready } \\
\text { for subsequent mounting of further } \\
\text { mechanical and electronical } \\
\text { devices }\end{array}$ \\
\hline
\end{tabular}

Table1. Generation process of optical systems

\begin{tabular}{|c|l|}
\hline $\begin{array}{c}\text { optical elements' } \\
\text { characteristics }\end{array}$ & \multicolumn{1}{c|}{ description } \\
\hline $\begin{array}{c}\text { commercial } \\
\text { requirements }\end{array}$ & maximum fabrication cost allowed and throughput to be produced \\
\hline geometry & $\begin{array}{l}\text { any combinations of flat, spherical, aspherical, off-axis aspherical or freeform } \\
\text { surfaces }\end{array}$ \\
\hline dimension & ranging from submillimeter to meter class optics \\
\hline quality & $\begin{array}{l}\text { described by parameters such as surface roughness, shape accuracy, centering } \\
\text { accuracy, power spectral density curve and material stress level }\end{array}$ \\
\hline
\end{tabular}

Table2. Classification of optical elements

\begin{tabular}{|c|c|c|l|l|l|}
\hline & $\begin{array}{l}\text { a) } \\
\text { brittle cracking }\end{array}$ & $\begin{array}{l}\text { b) } \\
\text { ductileFlow }\end{array}$ & $\begin{array}{l}\text { c) } \\
\text { chemical reaction }\end{array}$ & $\begin{array}{l}\text { d) } \\
\text { heat }\end{array}$ & $\begin{array}{l}\text { e) } \\
\text { sputtering }\end{array}$ \\
\hline - & $\begin{array}{c}S S D \text { cracks, } \\
\text { stress }\end{array}$ & $\begin{array}{c}\text { mechanical } \\
\text { stress, } \\
n\end{array}$ & $\begin{array}{c}\text { residuals, minerals } \\
\text { doped, environment, } \\
n, \text { "switch-off time» }\end{array}$ & $\begin{array}{c}S S D, \text { tension, } \\
\text { MSW, } n\end{array}$ & $\begin{array}{c}\text { dependency on } \\
\text { starting roughness, } \\
\text { very slow }\end{array}$ \\
\hline+ & fast & slow & uniform & $\begin{array}{c}\text { non-contact, } \\
\text { environmental } \\
\text { ly clean }\end{array}$ & $\begin{array}{c}\text { molekular accurate } \\
\text { shaping }\end{array}$ \\
\hline
\end{tabular}

Fig.3. Optical polishing techniques consist of combinations of five wear processes, each with its strong ("+" and weak ("“-") characteristics in terms of generating optical elements.

\begin{tabular}{|c|c|c|c|c|c|c|c|c|c|c|c|}
\hline $\begin{array}{c}\text { polishing } \\
\text { techniques }\end{array}$ & FFP & DG & $\mathbf{C P}$ & BFP & EEM & MRF & LP & IBF & ASJ & PACE & LIBWE \\
\hline $\begin{array}{c}\text { wear approaches } \\
\text { applicable } \\
\text { (see Fig.2) }\end{array}$ & $\begin{array}{l}\text { i, ii, } \\
\text { iii }\end{array}$ & i, ii & $\begin{array}{l}\text { ii, } \\
\text { v }\end{array}$ & $\begin{array}{l}\text { ii, iii, } \\
\text { v }\end{array}$ & $\begin{array}{l}\text { ii, iii, } \\
\text { v }\end{array}$ & iii & iv & $\begin{array}{l}\text { iii, } \\
\text { v }\end{array}$ & iii & iv, $\mathrm{v}$ & iv, v \\
\hline $\begin{array}{l}\text { based on these } \\
\text { combinations of } \\
\text { wear processes } \\
\text { (see Fig.3) }\end{array}$ & $\mathrm{b}, \mathrm{c}$ & $\mathrm{b}$ & $\mathrm{c}$ & $\mathrm{b}, \mathrm{c}$ & $b, c$ & $\mathrm{~b}, \mathrm{c}$ & $b, d$ & $\mathrm{e}$ & $\mathrm{b}, \mathrm{c}$ & $\mathrm{c}, \mathrm{d}$ & $\mathrm{c}, \mathrm{d}$ \\
\hline
\end{tabular}

Table3. classification of optical polishing techniques 


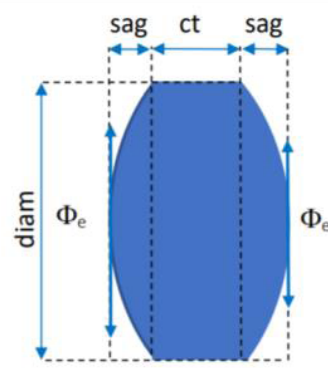

\begin{tabular}{|ll|}
\hline $\begin{array}{l}\text { LEFT SIDE (side 1) } \\
\text { clearAperture Фe: }\end{array}$ & 40 \\
prot.chamfer (length): & \\
coating (AR/special): & \\
3/power(irregularity) & $2(1)$ \\
$4 /$ (for flats use 3/) & 30 \\
$5 /$ & 0.064 \\
$6 /$ (if > 10J/cm^2 $=$ UDT) & $n 0$ \\
Sq surface roughness & 1.5 \\
smallest midSpatialWaelength: & 0 \\
sag & 6.7 \\
& YES \\
if spherical (YES/NO): & 50 \\
R: & \\
& \\
if aspherical (YES/NO): & \\
radius of removal sphere &
\end{tabular}

\begin{tabular}{|cl|}
\hline $\begin{array}{l}\text { MATERIAL: } \\
\text { if glass (YES/NO): }\end{array}$ & YES \\
HK: & 600 \\
AR: & SR1 \\
& \\
& \\
DIMENSIONS & \\
center thickness ct & 10 \\
ct tolerance & 0.05 \\
diameter & 50 \\
diam tolerance & 0.1 \\
& \\
& \\
PRODUCTION ORDER & \\
totalNumber PCS & 400 \\
batchSize pCS: & 40
\end{tabular}

\begin{tabular}{|c|c|}
\hline \multicolumn{2}{|l|}{ RIGHT SIDE (side 2) } \\
\hline $\begin{array}{l}\text { clearAperture Фe: } \\
\text { prot.chamfer (length): } \\
\text { coating (AR/special): }\end{array}$ & 45 \\
\hline $\begin{array}{l}\text { 3/power(irregularity) } \\
4 / \text { (for flats use } 3 / \text { ) }\end{array}$ & $1(0.5)$ \\
\hline $5 /$ & 0.064 \\
\hline $6 /($ if $>10 \mathrm{~J} / \mathrm{cm} \wedge 2=\mathrm{UDT}$ ) & no \\
\hline Sq surface roughness & 1 \\
\hline smallest midSpatialWaelength: & 0 \\
\hline sag & 4.3 \\
\hline if spherical (YES/NO): & YES \\
\hline $\mathrm{R}$ : & 75 \\
\hline $\begin{array}{l}\text { if aspherical (YES/NO): } \\
\text { radius of removal sphere }\end{array}$ & \\
\hline
\end{tabular}

Fig.3. Spherical biconvex lens made of glass.

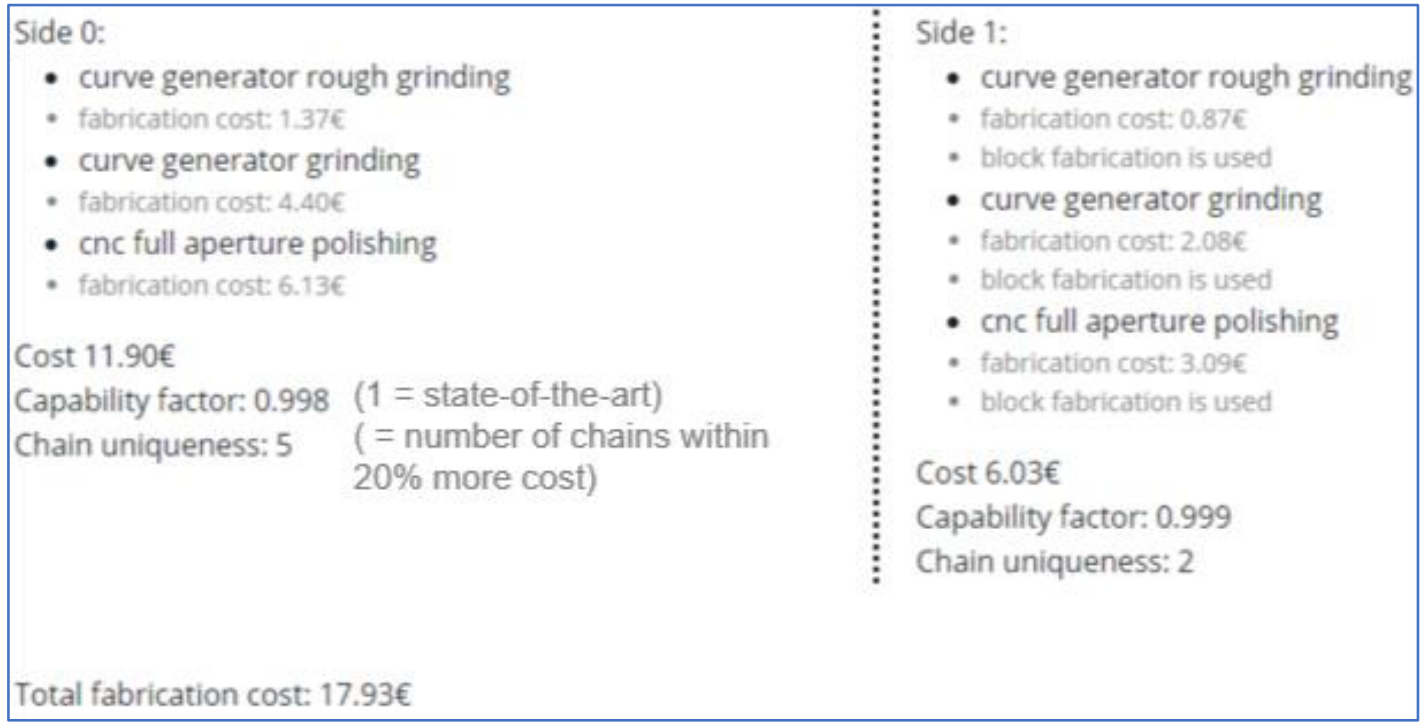

Fig.4. Modeling the generation of the biconvex lens shown in Fig.3: identification of the optimum optical fabrication technologies needed for the minimum-cost fabrication chain. 